\title{
Energy efficiency and performance limitations of linear adaptive control for transition delay
}

\author{
Nicolò Fabbiane ${ }^{1} \dagger$, Shervin Bagheri ${ }^{1}$ and Dan S. Henningson ${ }^{1}$ \\ ${ }^{1}$ Linné FLOW Centre, KTH Mechanics, S-10044 Stockholm, Sweden \\ (Received 23 May 2016; revised 14 September 2016; accepted 24 October 2016; \\ first published online 24 November 2016)
}

\begin{abstract}
A reactive control technique with localised actuators and sensors is used to delay the transition to turbulence in a flat-plate boundary-layer flow. Through extensive direct numerical simulations, it is shown that an adaptive technique, which computes the control law on-line, is able to significantly reduce skin-friction drag in the presence of random three-dimensional perturbation fields with linear and weakly nonlinear behaviour. An energy budget analysis is performed in order to assess the net energy saving capabilities of the linear control approach. When considering a model of the dielectric-barrier-discharge (DBD) plasma actuator, the energy spent to create appropriate actuation force inside the boundary layer is of the same order as the energy gained from reducing skin-friction drag. With a model of an ideal actuator a net energy gain of three orders of magnitude can be achieved by efficiently damping small-amplitude disturbances upstream. The energy analysis in this study thus provides an upper limit for what we can expect in terms of drag-reduction efficiency for linear control of transition as a means for drag reduction.
\end{abstract}

Key words: boundary layer control, drag reduction, instability control

\section{Introduction}

In low free-stream turbulence conditions, the transition to turbulence in a flat-plate boundary layer is dominated by Tollmien-Schlichting (TS) instabilities. These disturbances have the form of travelling waves that grow exponentially while propagating downstream. When they reach a critical amplitude, around $1 \%$ of the free-stream velocity, they nonlinearly interact with each other, eventually leading to a turbulent state. This scenario is known as the classical route to transition, as described in the review work by Kachanov (1994). Since a turbulent boundary layer leads to higher friction force, it is of engineering interest to develop control techniques that allow the flow to stay laminar as long as possible.

The general aim is to control the TS-waves instabilities when their amplitude is still small such as they reach the critical amplitude farther downstream. In this way, the nonlinear breakdown is used to our advantage; the disturbances are cancelled when their amplitude is low and the force requirement is small, where one can expect 
that the energy saving due to the drag reduction induced by the transition delay is very large. These considerations lead to an inherent - but not verified - high energy gain by this control strategy. Because of its potential, reactive flow control has been subjected to several studies in the past decades; the two-dimensional (2-D) control of flow instabilities has been widely investigated both from a numerical (e.g. Bagheri, Brandt \& Henningson 2009; Dadfar et al. 2013) and experimental (e.g. Kurz et al. 2013; Juillet, McKeon \& Schmid 2014; Kotsonis, Shukla \& Pröbsting 2015) point of view. Successful attempts to control complex 3-D environments can be found in the literature (Li \& Gaster 2006; Semeraro et al. 2013; Dadfar et al. 2014) but, to our knowledge, no systematic study on transition delay and energy saving has been conducted yet.

The present work aims to understand the transition-delay capabilities of reactive flow control and assess the potential net energy saving. In particular, the present work focuses on an adaptive control technique, which is based on an on-line computation of the control law. This is in contrast to static control techniques (Semeraro et al. 2013; Juillet et al. 2014), where the control law is precomputed, usually based on a model of the flow.

A multi-input multi-output (MIMO) filtered-x least-mean-squares (fxLMS) algorithm is used. This adaptive control technique has been studied by the experimental community and shown to be effective in 2-D TS-wave control (Sturzebecher \& Nitsche 2003; Kurz et al. 2013; Kotsonis et al. 2015). The fxLMS algorithm allows better stability and convergence with respect to conventional least-mean-squares (LMS) algorithms, when the error signal - i.e. the measurement of the cost function - is accessible only via a transfer function, called the secondary path (Ardekani \& Abdulla 2010). This is typical of the control of convective instabilities in feed-forward configuration, where the flow acts as a secondary path. In particular, a recent study by Fabbiane et al. (2015b) highlighted its robustness to varying external conditions when compared to static control. In particular, the algorithm was able to change the control law when the free-stream velocity was slightly varying from the nominal condition. The weak nonlinearities that TS-waves encounter in the first stages of the transition to turbulence can also be regarded as uncertainties; therefore, the algorithm should be able to adapt to the weak nonlinearities and extend the transition-delay capabilities of the investigated control set-up.

The manuscript is organised as follows: after a brief introduction to the numerical set-up $(\S 2)$ and the implemented adaptive algorithm $(\S 3)$, the control performances are investigated in linear $(\S 4.1)$ and nonlinear $(\S 4.2)$ regimes and transition-delay capabilities are analysed $(\S 4.3)$. Finally, the energy efficiency of reactive transition delay is evaluated $(\S 5)$ by using both ideal actuators and plasma-actuator models.

\section{Numerical simulations}

The incompressible Navier-Stokes equations govern the flow:

$$
\begin{gathered}
\frac{\partial \boldsymbol{u}}{\partial t}+(\boldsymbol{u} \cdot \nabla) \boldsymbol{u}=-\frac{\nabla p}{\rho}+\frac{1}{R e} \nabla^{2} \boldsymbol{u}+\lambda(\boldsymbol{x}) \boldsymbol{u}+\boldsymbol{f}, \\
\nabla \cdot \boldsymbol{u}=0,
\end{gathered}
$$

where $\rho$ is the density, $\boldsymbol{u}(\boldsymbol{x}, t)$ the velocity, $p(\boldsymbol{x}, t)$ the pressure at each time $t$ and position $\boldsymbol{x}=(X, Y, Z) \in \Omega$. The axis $X$ is aligned with the uniform and constant free-stream velocity $U_{\infty}, Y$ is normal to the surface and $Z$ defines a right-hand triad with the others, see figure 1 . A semi-infinite flat plate with infinitesimal thickness lies in the XZ-plane, on which a no-slip condition is enforced. Using a pseudo-spectral 


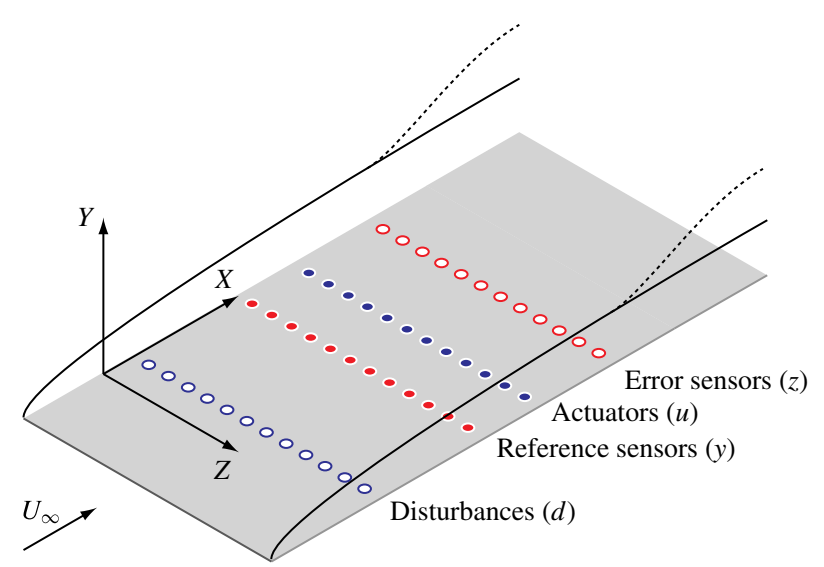

FIgure 1. (Colour online) Control set-up. Random 3-D disturbances are generated by a row of localised independent forcings $d$. The measurements from the sensors $y$ and $z$ are used by an adaptive fxLMS algorithm to compute the actuation signal for the actuators $u$ in order to reduce the amplitude of the detected disturbances.

code (Chevalier et al. 2007), direct numerical simulations (DNS) and large-eddy simulations (LES) are performed in order to analyse the control strategy. Periodicity is assumed in the spanwise and streamwise directions; the fringe forcing $\lambda(\boldsymbol{x})$ enforces periodicity in the streamwise direction in the last $20 \%$ of the streamwise domain length (Nordström, Nordin \& Henningson 1999). The volume forcing $f$ is used to introduce the disturbance and perform the control action, see $\S 2.1$. Spatial coordinates and velocities are non-dimensionalised by the displacement thickness in the beginning of the domain $\delta_{0}$ and the free-stream velocity $U_{\infty}$ respectively. The resulting Reynolds number is defined as $\operatorname{Re}=\delta_{0} U_{\infty} / v=1000$, where $v$ is the kinematic viscosity. For the time integration a fourth-order Crank-Nicholson/Runge-Kutta method is used with a constant time step $\Delta t=0.4$.

Two different computational domains are used in this work. A shorter domain $\Omega_{S}$ is used for the parametric study over the perturbation amplitude in $\S 4.2$. It extends for $[0,1000] \times[0,30] \times[-75,75]$ in the $X, Y$ and $Z$ directions and the flow is expanded over $1536 \times 384$ Fourier modes in the $X Z$-plane and 101 Chebyshev's polynomials in the wall-normal direction. A second and longer domain $\Omega_{L}$ is used to assess the transition delay and energy saving capabilities of the control technique in $\$ 4.3$. It extends for $[0,2000] \times[0,45] \times[-125,125]$ and it uses $1536 \times 151 \times 384$ Fourier-Chebyshev-Fourier basis. Dealiasing is performed along the Fourier-discretised direction with a $3 / 2$ rule.

Depending on the disturbance magnitude, turbulence may appear at the end of the longer domain. Since we are interested in the onset of turbulence and not in turbulence itself, a relaxation-term model (ADM-RT) is used as subgrid model (Schlatter, Stolz \& Kleiser 2004; Schlatter et al. 2010): in this way, we do not have to increase the spatial resolution in order to resolve the turbulent scales. This model has shown to be accurate and robust in predicting transitional flows (Schlatter et al. 2004).

\subsection{Inputs and outputs}

The input/output (I/O) set-up is composed of four rows of equispaced and independent objects (figure 1). Two rows of sensors $-y_{l}$ and $z_{l}$ - are placed at $X=300$ and 
$X=500$ : the former detects the upcoming disturbances and the latter measures the performance of the control action. The control is performed by a row of actuators $u_{l}$ positioned at $X=400$. These objects are positioned with a constant spanwise separation to cover the domain span. Semeraro et al. (2013) showed that a spanwise spacing $\Delta Z=10$ is necessary to effectively control a TS wavepacket for the current set-up; this results in 15 objects per row in the smaller domain $\Omega_{S}$ and 25 in the larger domain $\Omega_{L}$. The disturbances are introduced farther upstream at $X=65$ by a row of synthetic vortices $d_{l}$; the reference value for the disturbance is measured at $X=100$, where the perturbation is fully developed (see $\S 2.2$ ). Disturbance sources and actuators are modelled by the forcing term $f(X, Y, Z, t)$ in (2.1):

$$
\boldsymbol{f}=\boldsymbol{f}_{d}+\boldsymbol{f}_{u}=\sum_{l} \boldsymbol{b}_{d, l} d_{l}(t)+\sum_{l} \boldsymbol{b}_{u, l} u_{l}(t) .
$$

The constant spatial functions $\boldsymbol{b}_{d, l}(X, Y, Z)$ and $\boldsymbol{b}_{u, l}(X, Y, Z)$ are modulated by the disturbance and control signals $d_{l}(t)$ and $u_{l}(t)$, respectively.

Disturbance sources are modelled by localised synthetic vortices (Semeraro et al. 2013),

$$
\boldsymbol{b}_{d, l}=\left[\begin{array}{c}
\chi \tilde{Y} \\
-\gamma \tilde{X} \\
0
\end{array}\right] \exp \left(-\tilde{X}^{2}-\tilde{Y}^{2}-\tilde{Z}^{2}\right),
$$

where

$$
\tilde{X}=\frac{X-X_{d, l}}{\chi}, \quad \tilde{Y}=\frac{Y}{\gamma} \quad \text { and } \quad \tilde{Z}=\frac{Z-Z_{d, l}}{\zeta} .
$$

The $l$ th disturbance source is centred at $\left(X_{d, l}, 0, Z_{d, l}\right)$ and its spatial support is given by $\chi=2, \gamma=1.5$ and $\zeta=4$.

The control actuators are modelled as plasma actuators based on the experimental data by Kriegseis et al. (2013). This type of actuator has been adopted by Kurz et al. (2013), Fabbiane et al. (2015b), Kotsonis et al. (2015).

Following the work by Fabbiane et al. (2015b), localised measurement of the streamwise skin friction are used as sensors in order to model surface hot-wires (Sturzebecher \& Nitsche 2003; Li \& Gaster 2006; Kurz et al. 2013). Each signal is subtracted by its time average over 750 time units in order to remove the mean-flow contribution to the wall stress.

\subsection{Flow configurations}

Each disturbance source $d_{l}$ is independently fed with unitary uniform white noise $w_{l}(t)$,

$$
d_{l}(t)=a_{d} w_{l}(t)
$$

where the gain $a_{d}$ defines the amplitude. A uniformly distributed noise provides a better control of the maximum forcing amplitude that is fed to the flow, since the disturbance signal ranges between $\pm a_{d}$. Since the disturbance forcing in (2.4) is aligned with the streamwise direction and its spanwise component is zero, the resulting perturbation is dominated by the TS-wave.

Table 1 reports the flow configurations that are used in this work. The amplitude of the perturbation field is defined as:

$$
A^{2}(X)=\max _{Y}\left\langle\left(\frac{u^{\prime}}{U_{\infty}}\right)^{2}\right\rangle_{Z, t},
$$




\begin{tabular}{|c|c|c|c|c|}
\hline$a_{d}$ & $A(100)$ & $A(400)$ & Short box $\left(\Omega_{S}\right)$ & Long box $\left(\Omega_{L}\right)$ \\
\hline \multicolumn{5}{|c|}{ Linear behaviour } \\
\hline $1.0 \times 10^{-4}$ & $0.06 \times 10^{-3}$ & $0.01 \times 10^{-2}$ & S-LIN0 & - \\
\hline $1.0 \times 10^{-3}$ & $0.56 \times 10^{-3}$ & $0.09 \times 10^{-2}$ & S-LIN1 & L-LIN1 \\
\hline $2.0 \times 10^{-3}$ & $1.13 \times 10^{-3}$ & $0.19 \times 10^{-2}$ & S-LIN2 & L-LIN2 \\
\hline \multicolumn{5}{|c|}{ Weakly nonlinear behaviour } \\
\hline $3.0 \times 10^{-3}$ & $1.70 \times 10^{-3}$ & $0.33 \times 10^{-2}$ & S-WNL1 & L-WNL1 \\
\hline $4.0 \times 10^{-3}$ & $2.26 \times 10^{-3}$ & $0.55 \times 10^{-2}$ & S-WNL2 & L-WNL2 \\
\hline \multicolumn{5}{|c|}{ Nonlinear behaviour } \\
\hline $5.0 \times 10^{-3}$ & $2.83 \times 10^{-3}$ & $0.86 \times 10^{-2}$ & S-NL1 & L-NL1 \\
\hline $6.0 \times 10^{-3}$ & $3.40 \times 10^{-3}$ & $1.33 \times 10^{-2}$ & S-NL2 & L-NL2 \\
\hline $7.0 \times 10^{-3}$ & $3.98 \times 10^{-3}$ & $2.03 \times 10^{-2}$ & S-NL3 & L-NL3 \\
\hline \multicolumn{5}{|c|}{ Transitional behaviour } \\
\hline $8.0 \times 10^{-3}$ & $4.56 \times 10^{-3}$ & $2.97 \times 10^{-2}$ & S-TR1 & L-TR1 \\
\hline $9.0 \times 10^{-3}$ & $5.11 \times 10^{-3}$ & $4.00 \times 10^{-2}$ & - & L-TR2 \\
\hline $1.0 \times 10^{-2}$ & $5.68 \times 10^{-3}$ & $5.21 \times 10^{-2}$ & - & L-TR3 \\
\hline
\end{tabular}

TABLE 1. Flow configurations. The simulations are classified by the perturbation behaviour in the actuation region. The disturbance signals $d_{l}(t)$ are uniform white noises that range between $\pm a_{d}$. The amplitude $A(X)$ of the resulting perturbation field is reported close downstream of the disturbance source $(X=100)$ and at the actuators position $(X=400)$.

where $u^{\prime}$ is the streamwise component of the velocity with respect to the mean flow. The angled brackets $\langle\cdot\rangle$ indicate the average operator and the subscripts the averaging variables. In table 1 , the perturbation amplitude is reported at $X=100$, closely downstream to the disturbance-source location; a linear relation holds between the measured perturbation amplitude and the disturbance signal range $a_{d}$ for all the investigated flow cases. Hence, $A(100)$ is used to identify the introduced disturbance in the following.

The cases are grouped according to the perturbation behaviour at the actuators location $(X=400)$. In this study, three levels of nonlinear behaviour are identified. The flow is weakly nonlinear when the perturbation amplification deviate from the linear prediction but the control algorithm performance is not effected by the nonlinearity. Increasing the amplitude further, however, the adaptive algorithm is able to compensate only partially for the nonlinear behaviour of the flow; this scenario is thus identified as nonlinear. By increasing even further the disturbance amplitude, the laminar-to-turbulent transition reaches the actuation location and the control does not effectively control the perturbation field. The latter flow cases are transitional.

\section{Control strategy}

The control action is performed by a row of localised, equispaced actuators forcing the flow in the proximity of the wall. Their action $u_{l}(t)$ is computed based on the measurements $y_{m}(t)$ by a row of sensors upstream of the actuators: in this study, the number of sensors is equal to the number of actuators and they are aligned with the flow direction (figure 1).

A block diagram of the current set-up is shown in figure $2(a)$. The plant is the result of the interaction of the flow, sensors and actuators; it is the I/O description of 
(a)

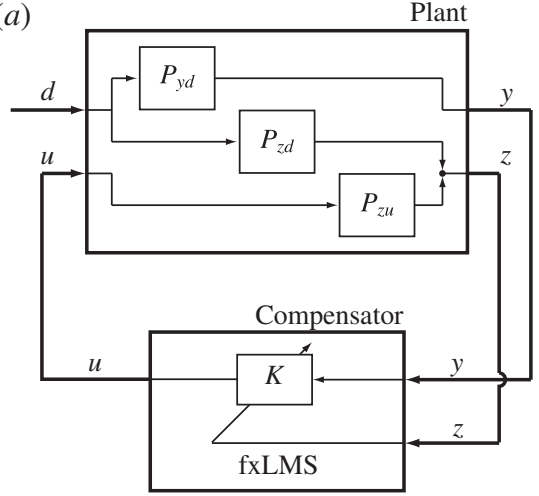

Block-diagram (b)

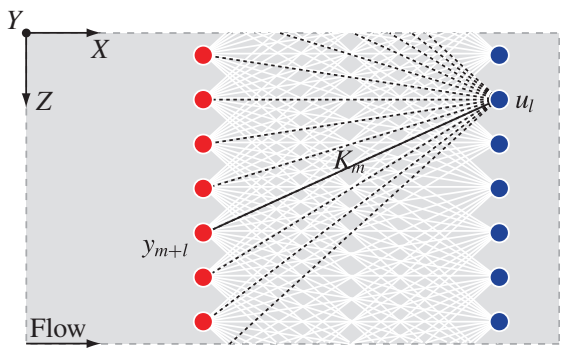

Control-law structure

FIgure 2. (Colour online) Compensator structure. The action of each actuator $u_{m}$ is computed by filtering the signals from all the sensor $y_{l}$ via a linear filter $K_{m}$. An adaptive fxLMS filter is responsible for computing the $K_{m}$ response in order to maximise in real time the control performances measured by the error sensors $z_{l}$.

the system that is meant to be controlled. In the linear approximation, the reference sensors signals $y_{l}$ are given by the disturbances $d_{l}$ filtered by the transfer function $P_{y d}$. The reference signal is fed to the compensator that computes the control action $u_{l}$ via the control law $K$. No contribution to $y_{l}$ comes from the control signals $u_{l}$ because the reference sensors are positioned upstream of the actuators and TS waves are convective instabilities. This leads to a feed-forward control strategy (Fabbiane et al. 2014). As shown by Belson et al. (2013), this configuration leads to better performance but it lacks robustness. Therefore, an adaptive method is used to create a closed loop on the control law via the performance outputs $z_{l}$ and recover robustness: this loop operates on a larger time scale than the control law $K$ and it recovers robustness for slow changes of the plant response (Fabbiane et al. 2015b).

We assume a linear control law and an equal number $(M+1)$ of sensors and actuators. As a consequence, the number of transfer functions between the $M+1$ sensors $y_{m}$ and the actuators $u_{l}$ is $(M+1)^{2}$. This imposes a computation constraint when $M+1$ is large, which is the case when covering a large spanwise width with the controller. However, since the flow is spanwise homogeneous, the same transfer $K_{m}$ function from all the sensors $y_{l}$ to one actuator can be replicated for each actuator $u_{m}$ (figure $2 b$ ). This assumption reduces the number of transfer functions to be designed from $(M+1)^{2}$ to $M+1$. The finite impulse response (FIR) filter representation of the control law reads,

$$
u_{l}(n)=\sum_{m=-M / 2}^{M / 2} \sum_{j=0}^{N} K_{m}(j) y_{m+l}(n-j),
$$

where $u_{l}(n)$ and $y_{l}(n)$ are the time-discrete control and measurement signals respectively, $K_{m}(j) \in \mathbb{R}^{(M+1) \times(N+1)}$ is the convolution kernel of the compensator and $N \Delta t$ is the time horizon of the FIR filter (Aström \& Wittenmark 1995).

The design of the compensator consists of computing the time-discrete convolution kernel $K_{m}(j)$. In this work, a MIMO version of the fxLMS algorithm is used to dynamically design the compensator (Sturzebecher \& Nitsche 2003; Fabbiane, 
Bagheri \& Henningson 2015a). The algorithm aims to minimise the sum of the squared measurement signals $z_{l}(n)$, i.e. the downstream row of sensors in figure 1 ,

$$
\min _{K_{m}}\left(\sum_{l=-M / 2}^{M / 2} z_{l}^{2}(n)\right) .
$$

The kernel is updated via a steepest descent algorithm at each time step,

$$
K_{m}(i \mid n+1)=K_{m}(i \mid n)-\mu \lambda_{m}(i \mid n),
$$

where the descend direction $\lambda_{m}(j \mid n)$ is given by

$$
\lambda_{m}(i \mid n)=\frac{\partial\left(\sum_{l} z_{l}^{2}(n)\right)}{\partial K_{m}(i)}=2 \sum_{l=-M / 2}^{M / 2} z_{l}(n) \frac{\partial z_{l}(n)}{\partial K_{m}(i)} .
$$

In order to compute the derivative in the previous equation, it is necessary to carry out the $z(n)$ dependencies by the control kernel $K_{m}(i)$. The error sensor signal is given by the superposition of the disturbance sources $d_{l}$ and actuators $u_{l}$,

$$
z_{l}(n)=z_{l, d}(n)+z_{l, u}(n) .
$$

Only the term $z_{l, u}$ depends on the control law $K_{m}(i)$ via the transfer function $P_{z u, r}(j)$,

$$
\begin{aligned}
z_{l, u}(n) & =\sum_{r} \sum_{j} P_{z u, r}(j) u_{r+l}(n-j) \\
& =\sum_{r} \sum_{j} P_{z u, r}(j) \sum_{m} \sum_{i} K_{m}(i) y_{m+r+l}(n-j-i) \\
& =\sum_{m} \sum_{i} K_{m}(i) \sum_{r} \sum_{j} P_{z u, r}(j) y_{r+m+l}(n-j-i) \\
& =\sum_{m} \sum_{i} K_{m}(i) f_{m+l}(n-i),
\end{aligned}
$$

where $f_{l}(n)=\sum_{r} \sum_{j} P_{z u, r}(j) y_{r+l}(n-j)$ are the filtered signals. For the sake of simplicity, the limit of the sums are omitted in (3.6): indices $r, l$ step from $-M / 2$ to $M / 2$ and $i, j$ from 0 to $N$. The same spanwise homogeneity assumption has been made for the plant kernel $P_{z u, r}(j)$, which represents the transfer functions $u_{r} \rightarrow z_{l}$. Hence the descent direction reads

$$
\lambda_{m}(i \mid n)=2 \sum_{l=-M / 2}^{M / 2} z_{l}(n) \frac{\partial z_{l}(n)}{\partial K_{m}(i)}=2 \sum_{l=-M / 2}^{M / 2} z_{l}(n) f_{m+l}(n-i) .
$$

Note that this method is not completely model free as $P_{z u, l+m}(i)$ is needed to compute $f_{l}(n)$. In this paper, this transfer function is computed via a linear impulse response of the actuator $u_{l}$. This transfer function is commonly addressed as the secondary path (Sturzebecher \& Nitsche 2003). The secondary path provides the control algorithm with information on how the actuators can affect the flow. In the current study the secondary path is obtained via a linear DNS of the impulsive response of one actuator. 


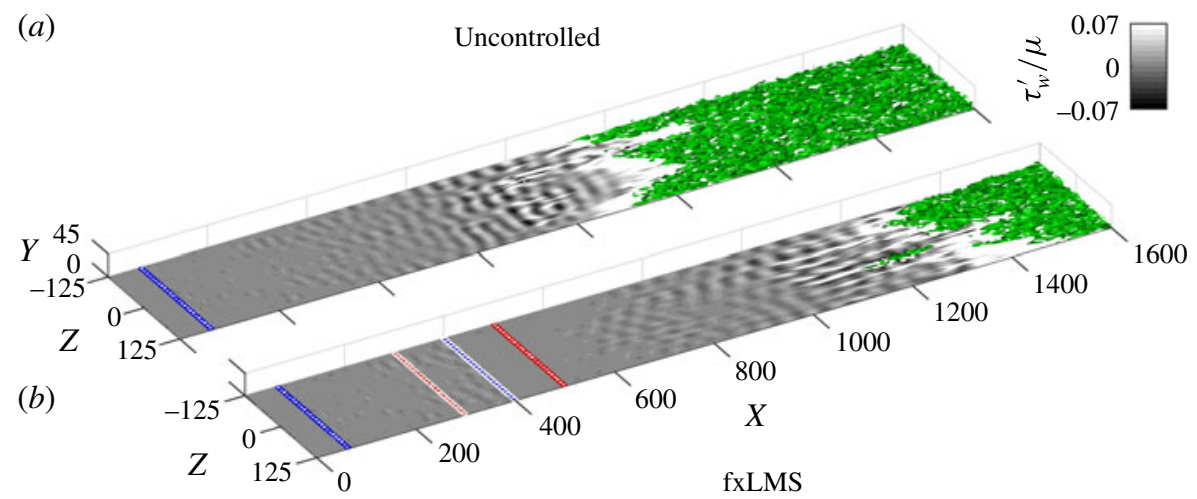

FIGURE 3. (Colour online) Disturbance attenuation and transition delay. The shaded grey area reports the skin-friction fluctuations $\tau_{w}^{\prime}$ (3.8). The green surfaces indicate the $\lambda_{2}$-criterion with a threshold of $-2 \times 10^{-3}$. The disturbance sources result in a perturbation field with amplitude $A(100)=0.11 \times 10^{-2}$ (case L-LIN2). The fringe region is not shown.

\subsection{The compensator in action}

The fxLMS algorithm is used to control randomly generated perturbations. Figure 3(a) shows the transition to turbulence in the uncontrolled case. The flow is perturbed with 25 disturbance sources; each one of these inputs is fed with an independent uniform white noise signal that ranges between $\pm 2 \times 10^{-3}$, (L-LIN2 in table 1). In figure 3, turbulent eddies are visualised by the $\lambda_{2}$-criterion in green (Jeong \& Hussain 1995); the disturbances grow and trigger transition in the second half of the domain. The grey shaded area shows the friction fluctuation $\tau^{\prime}(X, Z, t)$ at the wall with respect to the laminar solution:

$$
\tau_{w}^{\prime}=\tau_{w}-\tau_{w, l a m} .
$$

From the friction footprint, it can be seen that the disturbance sources create a random pattern of TS-wavepackets that grow while being convected downstream by the flow. When they reach a critical amplitude, they nonlinearly interact and trigger turbulence. The controlled configuration is shown in figure $3(b)$; the transition process is delayed, within the same disturbance environment. The disturbance amplitude drops downstream of the actuators (in blue) and the transition is significantly delayed with respect to the uncontrolled case. The step size $\mu$ is set equal to $10^{-5} / \sigma_{f}^{2}$, where $\sigma_{f}^{2}$ is the variance of the filtered signal $f_{l}(n)$.

The algorithm builds the control kernel $K_{m}(i)$ on-line based on the measurements upstream and downstream of the actuation region. In a low disturbance environment, the kernel will eventually converge to a steady solution; figure 4 shows the control kernel $K_{m}(i)$ for the presented simulation. The subscript $m$ is the spanwise shift between actuator $u_{l}$ and reference sensor $y_{m+l}$, hence it is directly related to the spanwise support of the control law. Its compact support in the spanwise direction indicates that the information given by the sensor is relevant only to compute the control signal for a limited number of actuators. This fact is related to the spanwise spreading of a wavepacket and shows how the control kernel is related to the structure of the disturbance that it is meant to control. The spanwise support of the control kernel is independent of the streamwise distance between sensors and actuators rows, as reported by Fabbiane et al. (2015a). 


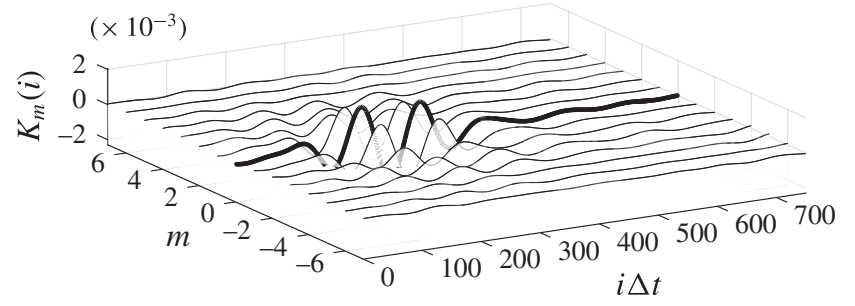

FIgURE 4. Control kernel. The thick line indicates $K_{0}(t)$, i.e. the connection between sensors and actuators at the same spanwise location.

\section{Control performance and limitations}

In this section, the performance of the control is analysed for small (\$4.1) and increasing magnitudes of the perturbation field $(\$ 4.2)$, up to the point where no transition delay and drag reduction are observed $(\$ 4.3)$.

\subsection{Linear control of linear perturbations}

In order to better understand how the compensator acts on the flow, the performance of the controller is studied when the perturbation field is small enough for its behaviour to be considered linear. For the flow case S-LIN0, the smaller computational domain $\Omega_{S}$ is used and the flow is perturbed by 15 disturbance source fed by 15 independent uniform white noise signals with amplitude $a_{d}=1.0 \times 10^{-4}$.

A Fourier transform is computed in time and in the spanwise direction. Hence a general flow quantity, e.g. the streamwise wall stress $\tau_{w}$, is transformed as:

$$
\hat{\tau}_{w}(X, \beta, \omega)=\left(\mathcal{F}_{z} \circ \mathcal{F}_{t}\right)\left(\tau_{w}(X, Z, t)\right),
$$

where $\beta$ and $\omega$ are the spanwise wavenumber and angular frequency, respectively. The temporal transform is based on 512 flow fields, 10 time units apart from each other; they are sampled after simulations reach statistical uniformity.

Figure 5(a,b) shows uncontrolled and controlled spectra for the skin friction $\hat{\tau}$ at the error sensor location, $X=500$. In the uncontrolled case, the disturbance field is present in a limited region of the spatio-temporal frequency space. The effect of the control is to damp the peak near to $(\omega, \beta) / 2 \pi=(0.01,0)$, as can be observed in figure $5(b)$.

The control also introduces some disturbances that are not present in the uncontrolled case, such as the double peak at $\beta / 2 \pi=0.1$ in figure $5(b)$. This perturbation is introduced by the actuators' spatial shape and spanwise distribution. These peaks are present for the superharmonics of the fundamental spanwise wavenumber of the actuator spacing $2 \pi / \Delta Z$. The actuator spacing is chosen according to Semeraro et al. (2013) in order to avoid these disturbances having support in the TS-wave region and, hence, interacting with the control action. Because of the limited amplitude and their short spanwise wavelength, they do not appear to compromise the control effect, also for higher disturbance levels.

The streamwise wall stress $\tau_{w}$ is a measurement of the disturbance at the surface. An integral measurement along the wall-normal direction is introduced to assess whether an overall reduction of the disturbance is correlated to a reduction of $\tau_{w}$ :

$$
A_{e}^{2}(X, \beta, \omega)=\int_{0}^{L_{Y}}|\hat{\boldsymbol{u}}(X, Y, \beta, \omega)|^{2} \mathrm{~d} Y,
$$




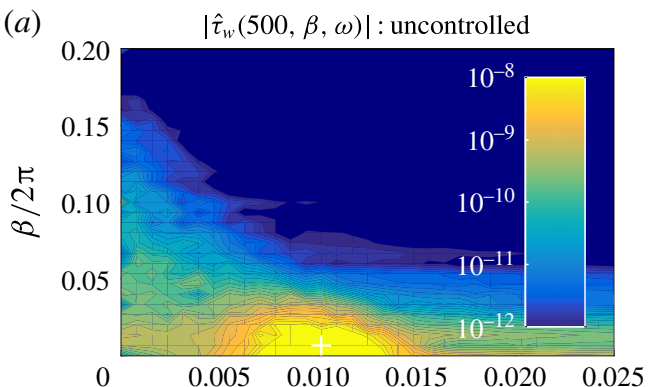

(b)
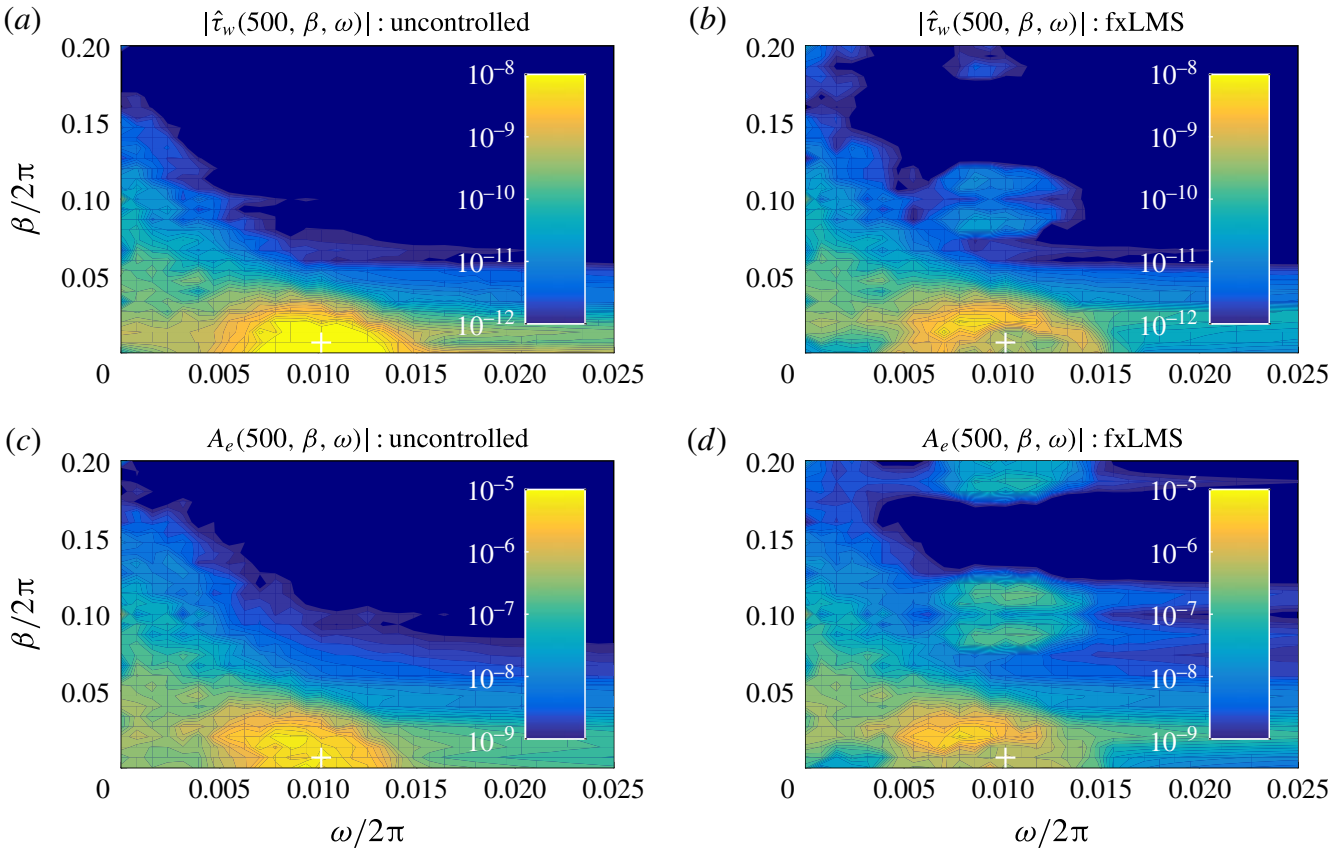

(d)

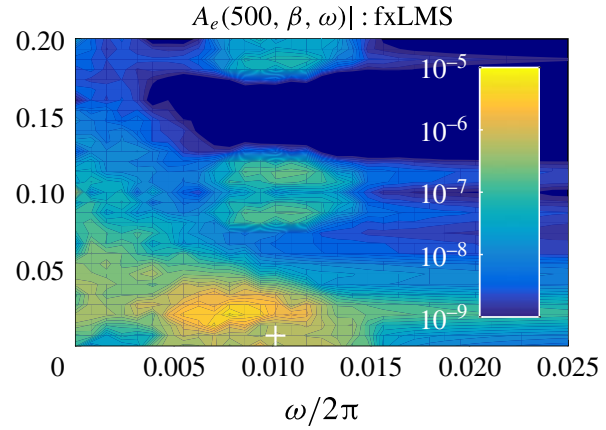

FIGURE 5. (Colour online) Control performance in low disturbance environment: $(a, b)$ depict the skin-friction spectra $\hat{\tau}$ for controlled and uncontrolled simulations at $z_{l}$-sensors location $X=500$, while $(c, d)$ report the energy-based amplitude $A_{e}$ at the same streamwise location. The spectra refer to the flow case S-LIN0, see table 1. The dark blue areas indicate values below the lower bound of the colour bar. The white cross indicates the Fourier mode reported in figure 6.

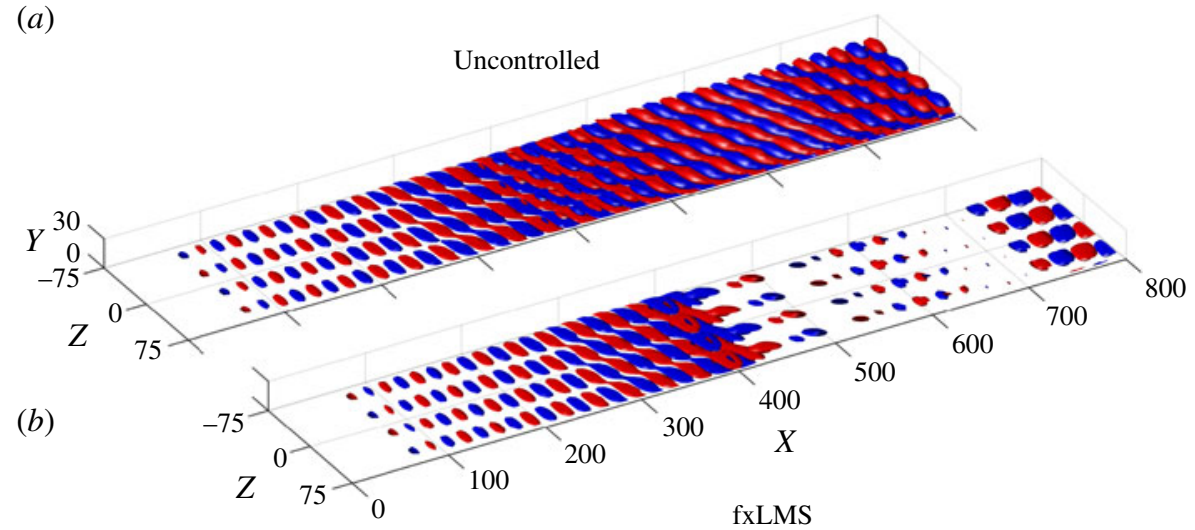

FIGURE 6. (Colour online) Fourier mode $\hat{\boldsymbol{u}}(X, Y, \beta, \omega)$ in physical space for $\omega / 2 \pi \approx 0.01$ and $\beta / 2 \pi=2 / L_{Z}$. Positive (red) and negative (blue) isosurfaces depict the real part of the streamwise velocity. The data refer to the same flow case as shown in figure 5 (S-LIN0).

where $\hat{\boldsymbol{u}}(X, Y, \beta, \omega)$ is the Fourier transform of the velocity $\boldsymbol{u}(X, Y, Z, t)$. Figure $5(c, d)$ reports $A_{e}$ for the controlled and uncontrolled case; both present the same features as the wall-stress spectra in figure $5(a, b)$. This shows that a reduction of the disturbance 
stress corresponds to a reduction of the disturbance energy; moreover, it confirms that the choice of measuring the disturbance amplitude by measuring its friction footprint is prudent.

Figure 6(a) shows a mode in the region of maximum amplification for the uncontrolled simulation. The mode has the appearance of a TS-wave triggered at the disturbance location and spatially growing throughout the domain. The effect of the control on the mode is clearly visible in figure $6(b)$; the perturbation grows until the actuator location $(X=400)$, after which it is cancelled almost completely within 100 spatial units. This confirms that the cancellation is not occurring suddenly at the actuator location. The actuator generates a counterphase wavepacket that cancels the original disturbance at the location of the error sensor. The cancellation is optimised at this streamwise position: downstream of this point, the disturbance and the control wavepacket continue to develop but the cancellation is not optimal, even if still effective. This explains why the disturbance slightly reappears downstream of the error sensor location before being convected out of the domain. A similar behaviour is common to all the Fourier modes in the damped region of the spectrum.

\subsection{The nonlinear challenge}

In this section, it is shown (i) how the linear control limits the performance of the investigated control strategy when nonlinearity is present and (ii) how adaptivity can reduce this performance loss. A parametric study over the perturbation amplitude is performed, where the 15 disturbance sources in $\Omega_{S}$ are fed with independent white noise signals of increasing amplitude.

Once the coupled compensator-flow system has reached the statistical steady state, the performance of the control action is tracked as a function of the disturbance level upstream of the actuation point. As introduced in $\S 2.1$, the sensors $y_{l}$ and $z_{l}$ measure wall-stress fluctuations, hence they are related to the amplitude of the perturbations at the sensing location. The amplitude measured by the error sensors is given by the measurement signals variance,

$$
\sigma_{z}^{2}=\frac{1}{M+1} \sum_{l=-M / 2}^{M / 2}\left\langle z_{l}(t)^{2}\right\rangle_{t},
$$

where each signal $z_{l}(t)$ has a zero temporal mean. The performance of the control action is assessed by the ratio between the controlled $\left(\sigma_{z, c}\right)$ and uncontrolled $\left(\sigma_{z, 0}\right)$ standard deviation of the error signals.

Figure 7 reports the performance indicator as a function of the perturbation amplitude $A(100)$. For perturbation amplitudes up to $0.11 \times 10^{-2}$, the control performance does not appear to be influenced by the disturbance amplitude. For higher amplitudes the control performance gradually departs form the linear behaviour, as the nonlinearities start to become relevant at the actuator position. Figure 8 reports $A(X)$ at the actuator location $X=400$ for the uncontrolled case; the perturbation behaves nonlinearly when $A(100)$ is greater than $\sim 0.17 \times 10^{-2}$. Comparing with figure 7 , it is clear that the performance loss of the control strategy is related to the rise of nonlinearities in the flow.

The adaptivity properties of the fxLMS algorithm are favourable when it comes to slowly varying conditions in the flow (Fabbiane et al. 2015b). However, when it comes to nonlinearities, they are only capable of a marginal improvement of the control performance. As introduced in $\S 3$, the fxLMS algorithm acts on the control 


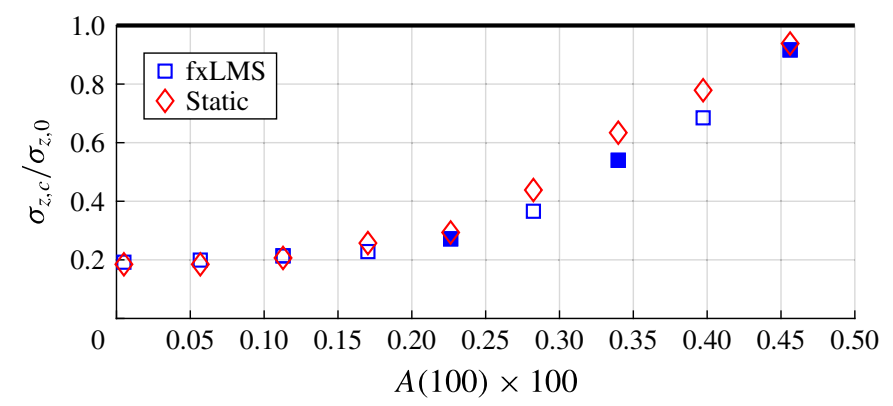

Figure 7. (Colour online) Performance indicator $\sigma_{z, c} / \sigma_{z, 0}$ as a function of the seeded perturbation amplitude $A(100)$. The filled markers indicate the flow cases reported in figures 9 and 10.

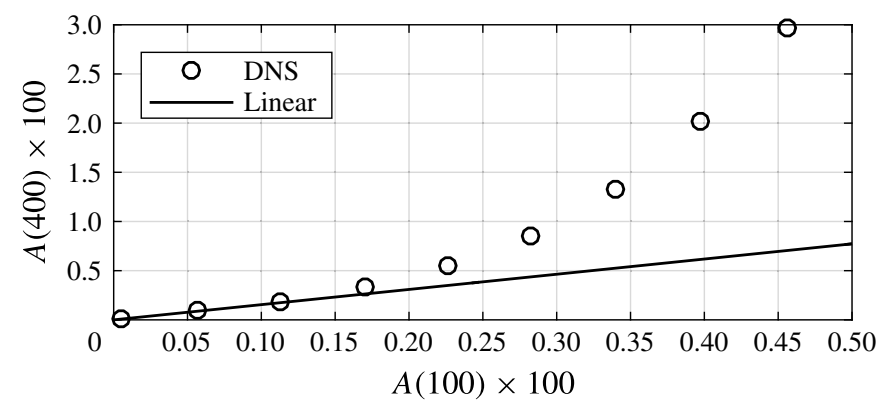

FIGURE 8. Perturbation amplitude $A(400)$ at the location of the actuators as a function of the perturbation amplitude $A(100)$.

law by changing the control kernel $K_{m}(i)$ according to the measurement from the error sensors. The role of the adaptivity in controlling nonlinear flows is highlighted by comparing the adaptive fxLMS algorithm to a static control law, where the adaptive fxLMS algorithm is switched off. The red diamond symbols in figure 7 report the control performance when the static control law is considered.

The gradual loss of performance by the compensator can be analysed by studying the wall-friction spectra at the error sensor location; figure 9 shows the uncontrolled and controlled spectra for increasing disturbance amplitudes, while figure 10 reports instantaneous flow fields for the same simulation parameters. For the lowest reported amplitude, the flow has a linear behaviour. TS-waves start to nonlinearly interact with themselves and generate the structures close to the $\beta$-axis; this is visible both for the uncontrolled and control cases.

As the amplitude increases (figures $9 a, b$ and 10a) disturbances arise around $(\omega, \beta) / 2 \pi \approx(0.005,0.075)$; the amplitude of these modes in the controlled case is lower than in the uncontrolled one. By cancelling the perturbation in the TS-wave region, the control is able to delay the growth of the secondary disturbances that will eventually lead to turbulence.

Figures $9(c, d)$ and $10(b)$ show the limit amplitude for which the control has an effect on the perturbation field. The peak related to the TS-wave is still damped but the modes due to nonlinear interactions of the perturbation field are clearly visible in both cases; the perturbation behaviour is already nonlinear at the actuation location 


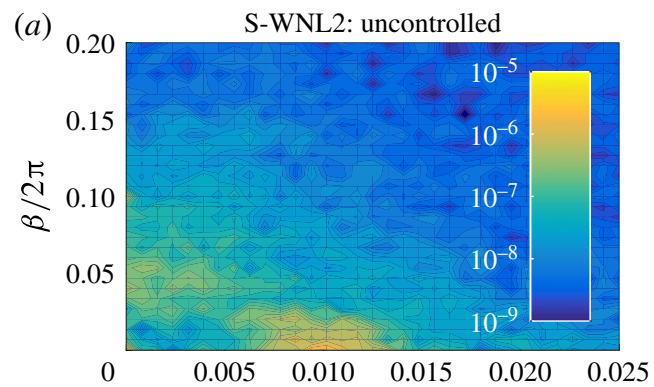

(b)
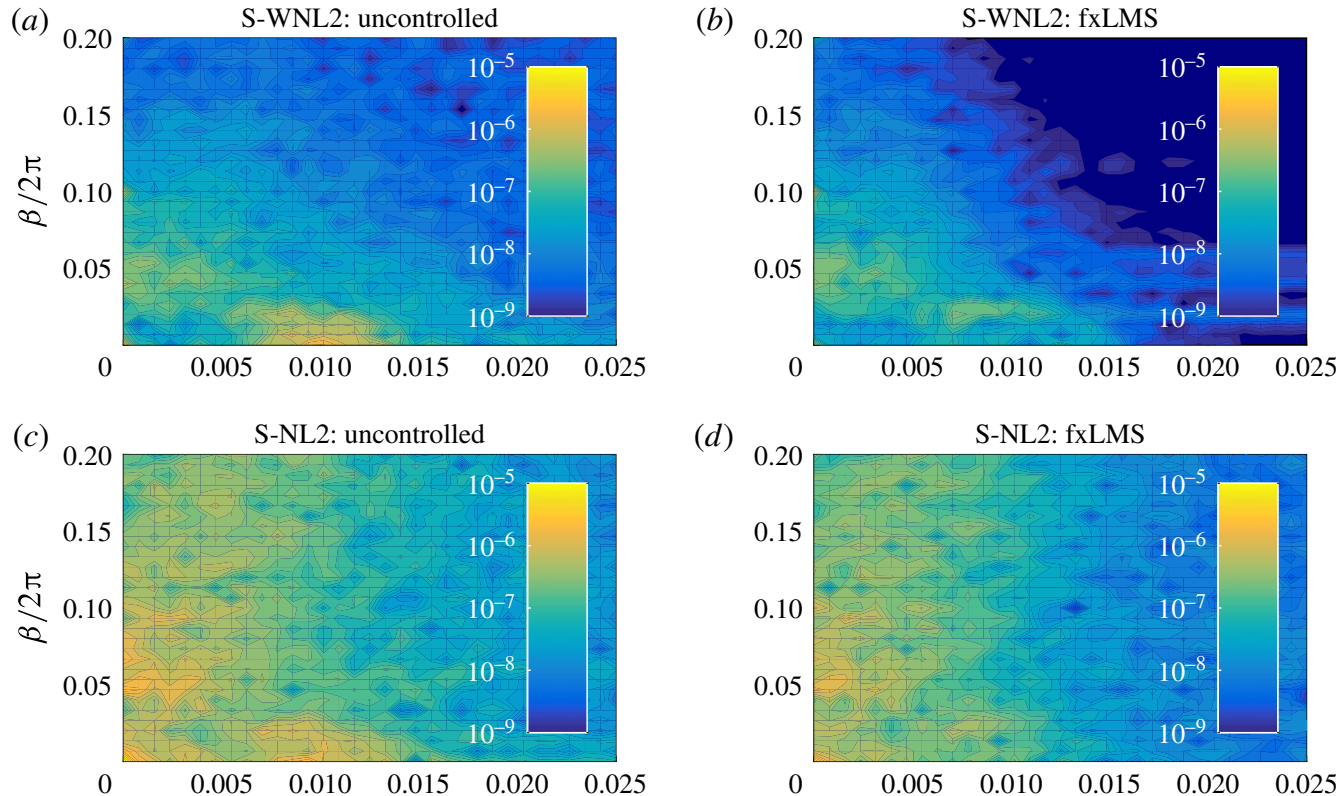

(d)
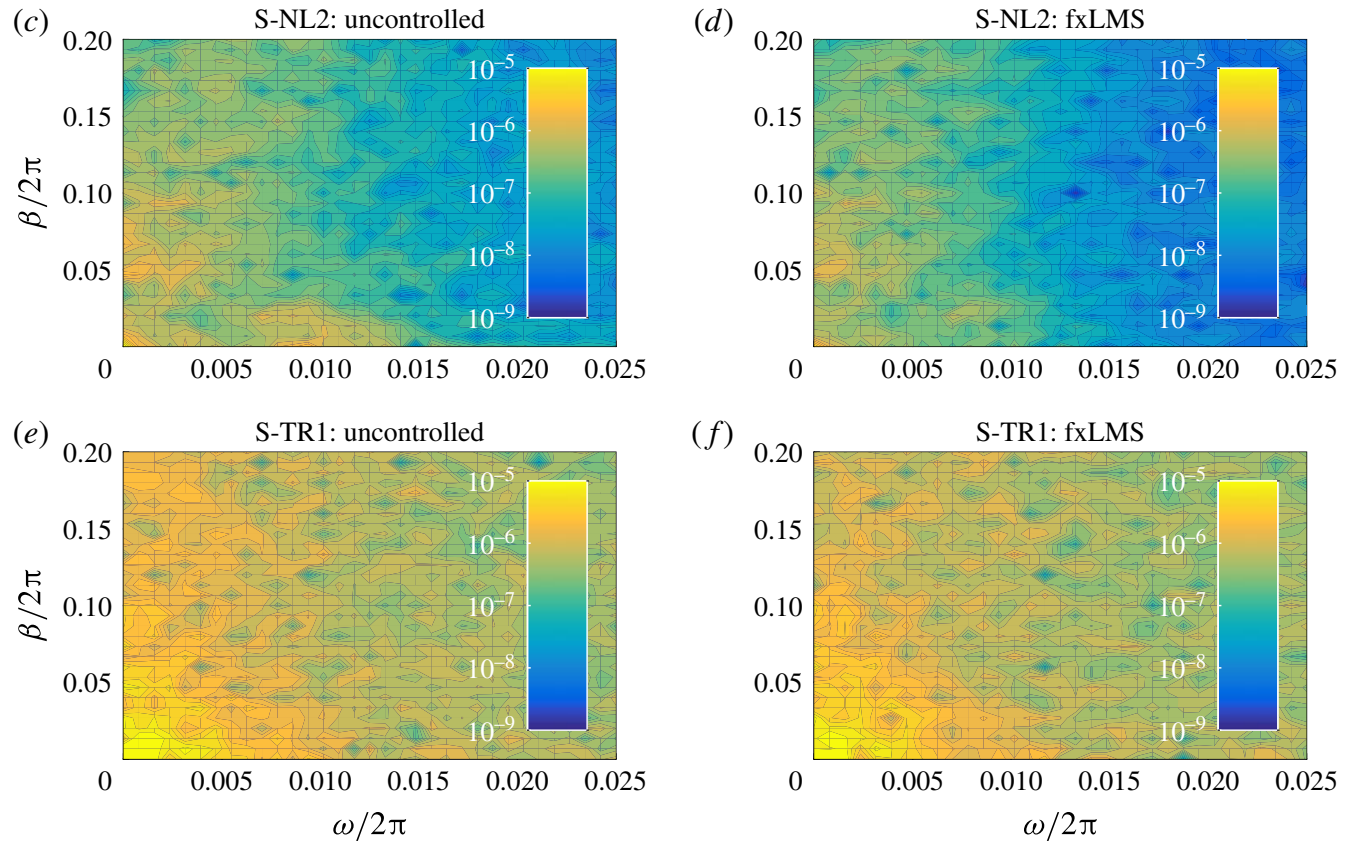

FIgURE 9. (Colour online) Skin-friction spectra for uncontrolled and controlled simulations at $z_{l}$-sensors location $X=500$. The disturbance sources produce a perturbation field with amplitude $A(100)$ as in the subcaption of the figures. The dark blue areas indicate values below the lower bound of the colour bar.

(see figure 8). Finally, in figures $9(e, f)$ and $10(c)$ the uncontrolled and controlled simulations are almost undistinguishable; for this amplitude, transition to turbulence will take place just downstream of the error sensor location and no transition delay is noticeable, see $\S 4.3$.

In all the presented scenarios it is observed that the control is able to directly damp only disturbances in the TS-wave region. This is explained by the fact that the algorithm uses a linear model of the flow - the secondary path $P_{z u, m}$ - to identify the control law. The model is unable to capture the nonlinear interactions of the perturbation field and, hence, the control action focuses on the linear mechanism in the flow.

Figure 11 shows the converged control kernel connection $K_{0}(i)$ between sensors and actuators with the same spanwise location, for three different disturbance amplitudes. Adaptive effects appear when the nonlinearities arise in the flow. In this range of 

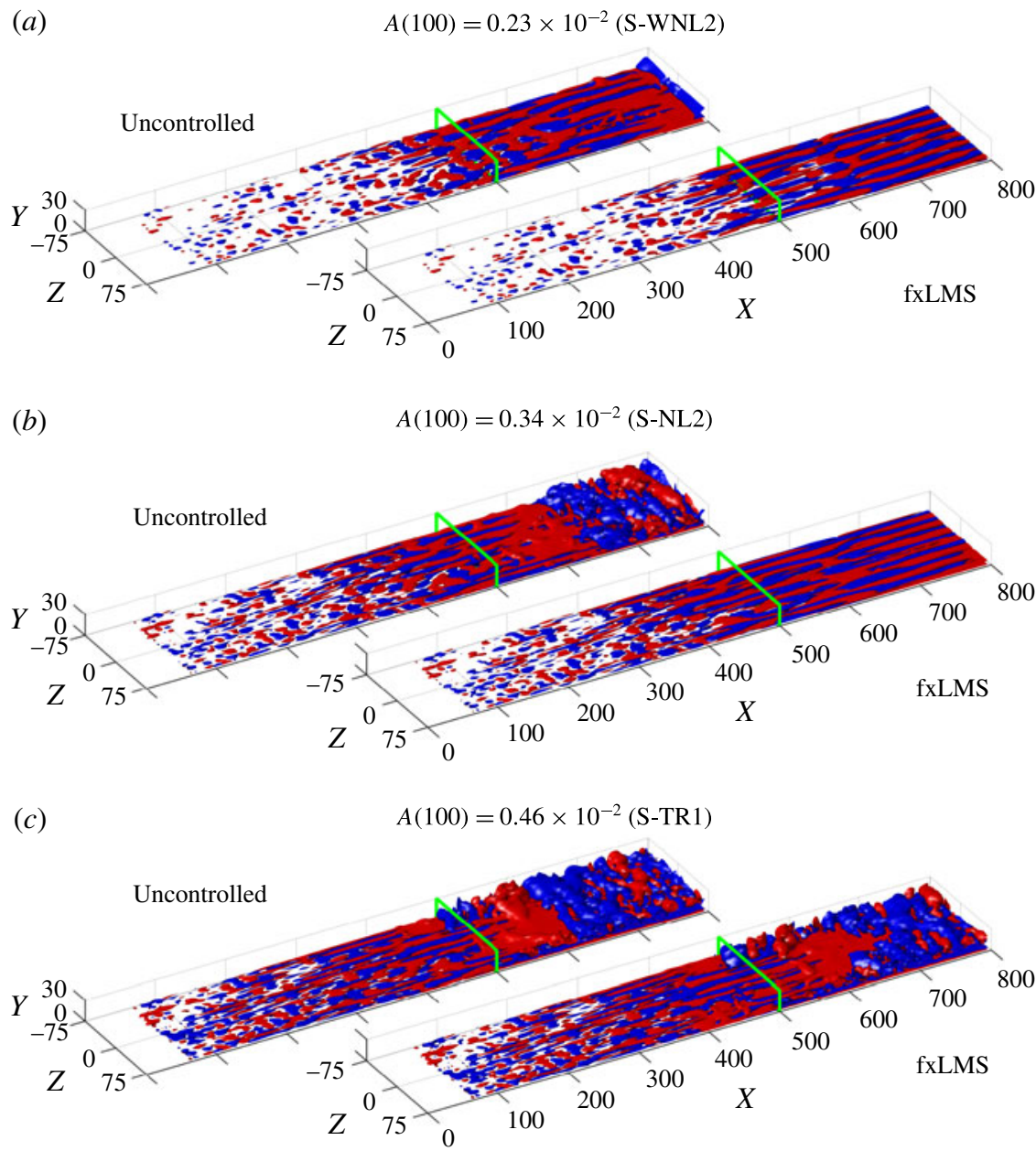

FIGURE 10. (Colour online) Velocity fluctuations isosurfaces $u^{\prime}$ with respect to the laminar solution for increasing disturbance amplitude. Red and blue isosurfaces are obtained for $u^{\prime} / U_{\infty}= \pm 0.25 \%$. The green plane indicates the streamwise position $(X=500)$ where the spectra in figure 9 are computed.

amplitudes, the fxLMS solution show slightly better performance with respect to the static controller. At this point the nonlinearities are weak and their effect is limited to a change in the amplification and phase shift of the travelling waves. The algorithm modifies the control kernel by increasing the gain and reducing the time shift between sensors and control signals (dashed line in figure 11). However, the adaptive capabilities of the algorithm have a limit. Since the nonlinear flow modification also has an effect on the input/output behaviour of the system, the secondary path model used by the algorithm is no longer consistent with the real secondary path in the flow. The algorithm is able to compensate this error if the phase difference between real and modelled secondary path is lower than $\pi / 2$ in absolute value (Snyder \& Hansen 1994; Simon et al. 2015). Hence, the control will continue to reduce the amplitude of the disturbances modelled by the secondary path, 


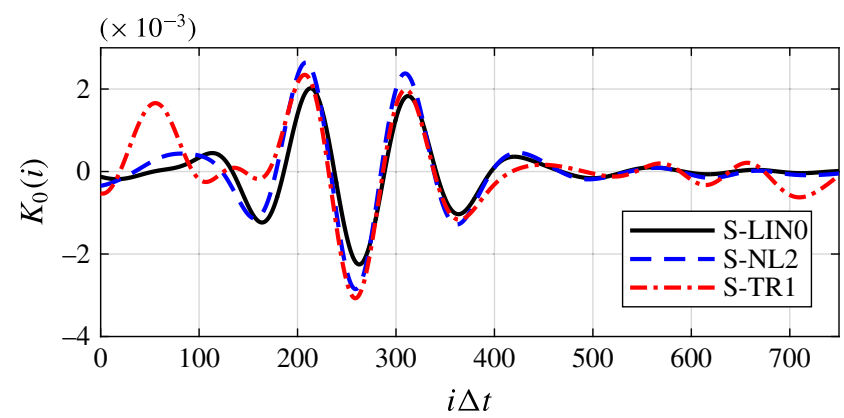

Figure 11. (Colour online) Comparison of the control kernels $K_{0}(i)$ for three different disturbance levels. The kernel for the case S-LIN0 is also the static control law in figure 7.

up to the point where the phase error caused by the nonlinearities in the flow is large enough to destabilise the fxLMS algorithm.

At this point, the performance margin given by the adaptivity with respect to the static control tends to zero. This occurs when the transition is incipient in the region of the flow where the control action takes place, as seen in figure $9(g-h)$. The dotdashed line in figure 11 shows the control kernel in this scenario; the flow is already transitional at the actuator location and the adaptive algorithm introduces non-physical solutions of the control kernel.

\subsection{Transition delay}

It has been shown in the previous section that the control is able to reduce the perturbation amplitude downstream of the actuators. This section analyses how this disturbance reduction translates into a transition delay. The long box $\Omega_{L}$ is used to assess where the transition to turbulence occurs both in uncontrolled and controlled cases.

Delaying the laminar-turbulent transition means extending the portion of the flow that is laminar, which results in a lower total skin friction. Figure 12(a) shows the spanwise-averaged friction coefficient, defined as

$$
c_{f}(X)=\frac{\left\langle\tau_{w}\right\rangle_{Z}}{\frac{1}{2} \rho U_{\infty}^{2}},
$$

corresponding to the flow shown in figure 3. The friction rise related to the onset of the turbulent regime is clearly delayed and the laminar friction region is extended in the controlled case.

The transition location is identified as the point where the average friction in the flow crosses the average between the laminar solution and the turbulent value as predicted by the Schultz-Grunow formula (Schultz-Grunow 1940). The transition location moves upstream as the disturbance level increases (figure 12b). The perturbation amplitude reduction, which the control is capable of, leads to a transition delay for all investigated disturbance levels. However, the delay reduces as the amplitude increases and the disturbance reduction becomes less effective.

The green diamond symbols in figure $12(b)$ report the transition location when the error sensor is displaced downstream by 100 spatial units. A performance loss is observed for lower amplitude than the original set-up; this shows that the 
(a)

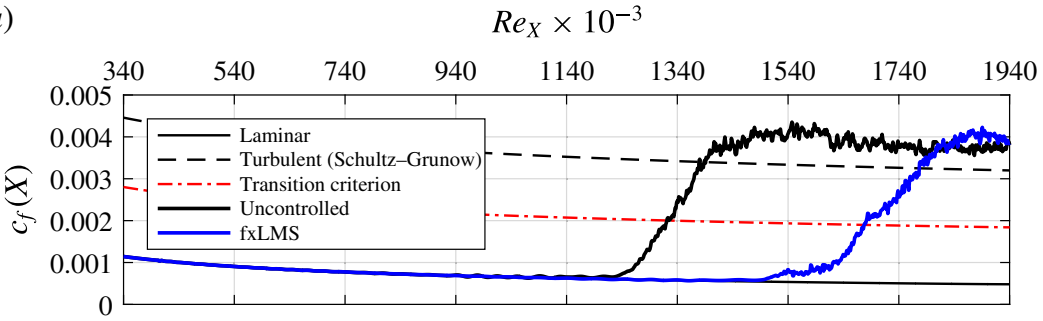

(b)

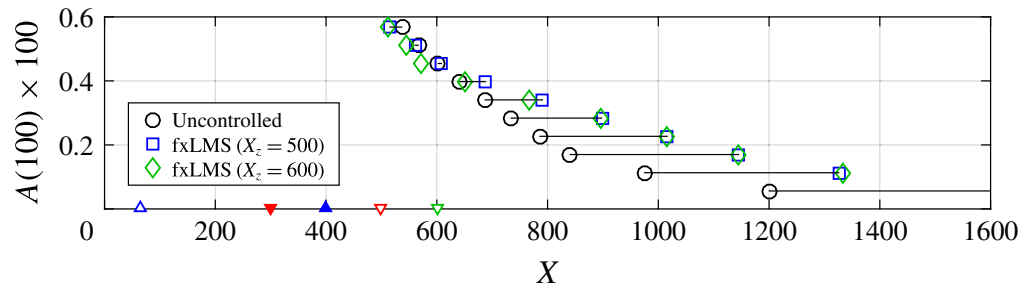

FIGURE 12. (Colour online) Transition delay: (a) reports the spanwise-averaged friction coefficient for the flow case in figure 3, L-LIN2; $(b)$ reports the transition location for increasing perturbation amplitude $A(100)$; the reported positions are computed based on a time-averaged flow over 1200 time units. The top axis reports $\operatorname{Re}_{X}=\left(X-X_{l e}\right) U_{\infty} / v$, where $X_{l e}$ is the extrapolated leading edge position according to Blasius boundary-layer solution.

performance limit is given by the disturbance amplitude at the error sensor location and not at the actuator location. This is in contrast with the linear analysis by Fabbiane et al. (2015a), where they show that better performance is obtained when the error sensors are far from the actuators.

The transition delay results in a drag reduction. The amount of saved drag is given by the area between the controlled and uncontrolled curves in figure 12(a):

$$
\frac{\Delta D}{L_{Z}}=\int_{0}^{L_{X}}\left\langle\tau_{w, 0}-\tau_{w, c}\right\rangle_{Z} \mathrm{~d} X,
$$

where $\tau_{w, 0}$ is the wall shear stress in the uncontrolled case and $\tau_{w, c}$ in the controlled one. By repeating the same procedure for the different disturbance amplitudes in figure $12(b)$, the drag reduction as a function of the perturbation level is shown in figure 13.

For the higher amplitudes the transition location approaches the region where the actuation takes place; as shown in the previous section, the nonlinearities that eventually lead to transition start to develop at the error sensor location, which reduces the control capabilities of the algorithm. Hence, the investigated control technique is effective in delaying the laminar-to-turbulence transition when the perturbation amplitude at the actuation location is lower than $2 \%$ of the free-stream velocity, according to the amplitude definition in (2.7). For higher amplitudes, the control is not able to delay the already incipient transition; the strong nonlinear behaviour of the flow inhibits the adaptive algorithm to converge to an effective control law. This introduces eventually disturbances that shorten the transition region and, as a consequence, leads to the drag increase as shown in figure 13. 


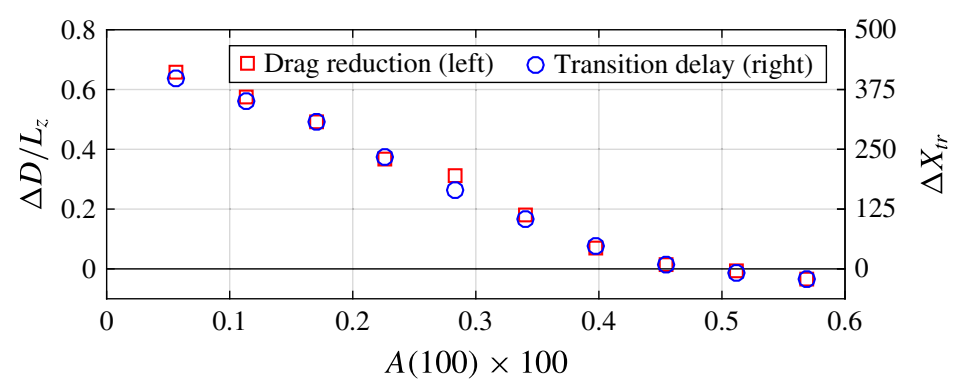

FIgURE 13. (Colour online) Drag save and transition delay as a function of the perturbation level $A(100)$. The reported quantities are based on a time-averaged flow over 1200 time units.

\section{Energy efficiency}

In the previous section it was shown that the investigated control strategy is able to delay the transition to turbulence and consequently reduce the friction drag. In this section, ideal and real actuator models are introduced in order to assess the energy efficiency of this control technique. To the best of our knowledge, this is the first time that the energy gain given by reactive laminar flow control techniques is assessed in a systematic manner.

\subsection{Actuator models}

Actuator models are introduced in order to compute the consumed power by the actuators in order to perform the control action. An ideal actuator is introduced in order to assess the theoretical energy gain and then compared with a more realistic experimental model of plasma actuators.

The ideal actuator is based on the volume integral across the domain of the local power $\left(\boldsymbol{u} \cdot \rho \boldsymbol{f}_{u}\right)$ exchanged between the flow and the volume forcing:

$$
P_{c, i}=\left\langle\left|\int_{\Omega} \boldsymbol{u} \cdot \rho \boldsymbol{f}_{u} \mathrm{~d} \Omega\right|\right\rangle_{t} .
$$

This actuator model cannot extract power from the flow; both positive and negative integral values are power consumption. Therefore, the definition of power is based on the magnitude of the local power. A similar approach is used when it comes to blowing/suction actuators (e.g. Stroh, Frohnapfel \& Schlatter 2015), where the time average of the absolute value of the instantaneous power needed to enforce the mass flux is used to compute the used power by the control technique.

As introduced in $\$ 2.1$, a DBD plasma actuator is considered as a model for the actuator volume forcing. In particular, the work by Kriegseis et al. (2013) is used, where the plasma-actuator force field is reconstructed starting from particle image velocimetry (PIV) flow measurements. Based on their measurement it is possible to correlate the AC voltage supply $V_{p}$ and the provided force $F / L$. As reported in figure 14(a), the voltage-force relation can be well represented by the linear regression:

$$
V_{p}=\Phi \frac{F}{L}+V_{0}
$$




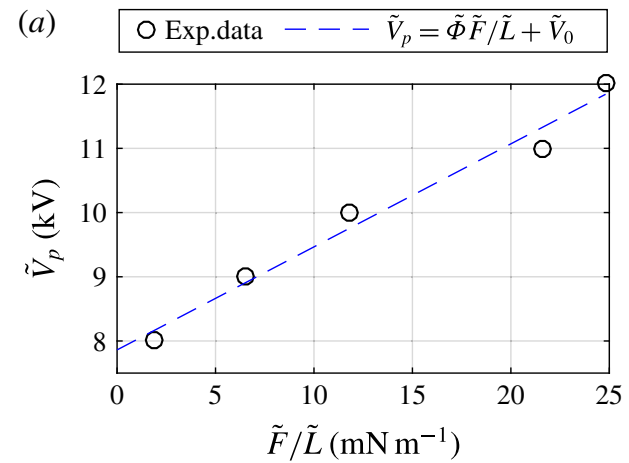

Force (Kriegseis et al. 2013)

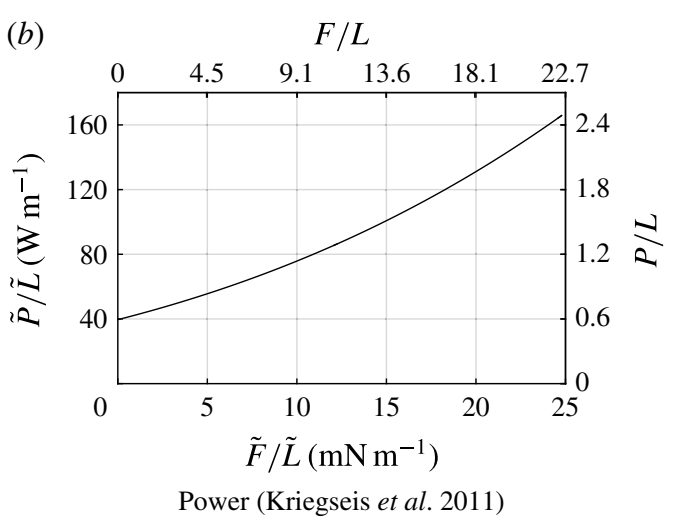

FIgURE 14. (Colour online) Plasma-actuator contsitutive laws. The tilde indicates dimensional quantities.

where $L$ is the spanwise length of the actuator and $\Phi$ and $V_{0}$ are ad-hoc coefficients. In particular, the latter indicates the voltage for which the plasma actuator is giving zero force and can be considered as a lower limit for the supplied voltage. In fact, the plasma actuator is not capable of supplying a negative force; in order to overcome this issue, two different operation modes are typically considered.

(i) Dual mode: two plasma actuator facing each other are considered for each actuation station $u_{l}$. One is responsible for the positive part of the actuation signal and one is responsible for the negative one.

(ii) Hybrid mode: a single plasma actuator is considered. An offset is applied to the voltage in order not to cross the zero-forcing voltage $V_{0}$; the offset depends on the minimum amplitude of the control signal $u_{l}(t)$ in the averaging window. The constant forcing that results from the offset has a stabilising effect on the boundary layer (Kurz et al. 2013); in the present study this effect is not taken into account.

Once the operation mode is defined, the power used by the actuator is estimated via the relation proposed by Kriegseis et al. (2011):

$$
\frac{P}{L}=\Theta \sqrt{V_{p}^{7} f_{p}^{3}}=\Theta \sqrt{\left(\Phi \frac{F}{L}+V_{0}\right)^{7} f_{p}^{3}},
$$

where $f_{p}$ is the plasma-actuator AC-supply frequency. The coefficient $\Theta$ is found to be an almost universal coefficient equal to $5 \times 10^{-4} \mathrm{~W} \mathrm{~m}^{-1}(\mathrm{kHz})^{-3 / 2}(\mathrm{kV})^{-7 / 2}$ (Kriegseis et al. 2011). For the current case a dimensional supply frequency $\tilde{f}_{p}=15 \mathrm{kHz}$ is considered. All the quantities in (5.3) are non-dimensionalised by considering kinematic viscosity $\tilde{v}=1.5 \times 10^{-5} \mathrm{~m}^{2} \mathrm{~s}^{-1}$, free-stream velocity $\tilde{U}_{\infty}=60 \mathrm{~m} \mathrm{~s}^{-1}$, density $\tilde{\rho}=1.225 \mathrm{~kg} \mathrm{~m}^{-3}$ and Reynolds number $R e=\tilde{U}_{\infty} \tilde{L} / \tilde{v}=1000$ as in the simulations, see $\S 2$.

The force $(F / L)_{l}$ required by each actuator can be computed by knowing the control signal $u_{l}(t)$ and its forcing shape $\boldsymbol{b}_{u, l}$ from (2.3). Since the control forcing is time dependent, the time-averaged power is considered to evaluate the power consumption of the actuator. Hence, the individual power consumption $(P / L)_{l}$ is computed and the 


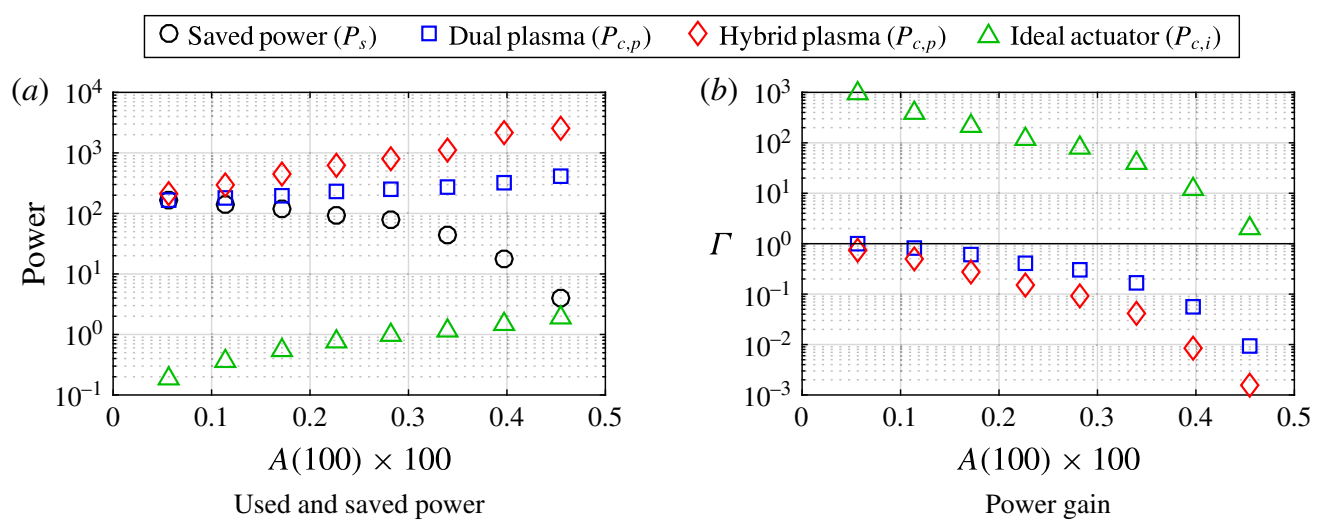

FIGURE 15. (Colour online) Energy budget. The reported quantities are computed based on a time-averaged flow over 1200 time units. The solid black line in $(b)$ indicates $\Gamma=1$, i.e. the break-even point for the control strategy.

total power consumption $P_{c, p}$ is estimated by summing the time-averaged contribution of each actuator:

$$
P_{c, p}=\sum_{l=-M / 2}^{M / 2}\left\langle\left(\frac{P}{L}(t)\right)_{l} \Delta Z\right\rangle_{t},
$$

where $\Delta Z$ is the spanwise support of the actuator.

\subsection{Power gain}

The saved power is quantified by the product of the drag reduction $\Delta D$ and the freestream velocity $U_{\infty}$ (Stroh et al. 2015):

$$
P_{s}=U_{\infty} \Delta D
$$

In figure 15(a) $P_{s}$ is compared with the power used by the actuators computed via the different actuator models. Ideal and plasma actuator show similar trends with increasing disturbance level; they consume more power as the disturbance amplitude becomes larger. On the other hand, the saved power reduces because of the control performance loss due to the nonlinearities at the actuation location.

The power-gain coefficient is defined as:

$$
\Gamma=\frac{P_{s}}{P_{c}}
$$

This coefficient gives the saved power because of the transition delay as fraction of power $P_{c}$ invested in the control. The break-even point is given by $\Gamma=1$, when the energy that is spent for the control is equal to the saved energy $P_{s}$.

For the ideal actuator, a theoretical gain between $10^{3}$ and $10^{2}$ is possible for perturbation amplitude of the order of few per cent (figure 15b). For larger disturbance amplitudes, the gain gradually decays and eventually crosses the break-even point.

The energy gain based on the plasma-actuator power-consumption estimation is lower than the break-even value for all the investigated cases. In order to better 


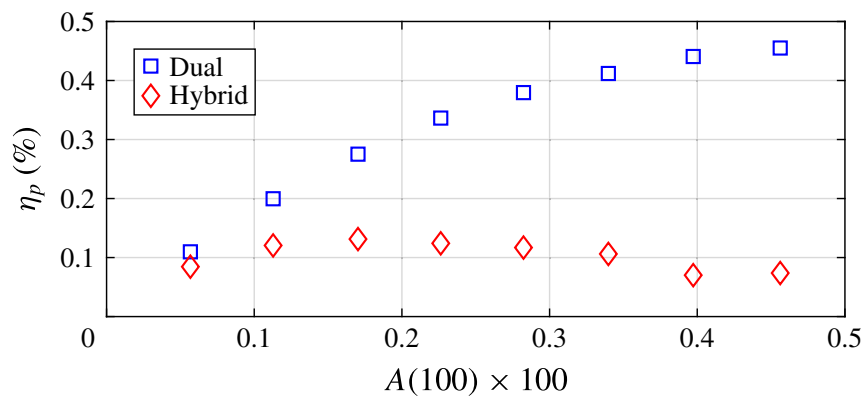

FIGURE 16. (Colour online) Plasma-actuator efficiency $\eta$ with respect to the ideal actuator.

compare it to the ideal-actuator model, let us introduce a measurement of the actuator efficiency:

$$
\eta_{p}=\frac{P_{c, i}}{P_{c, p}}=\frac{\Gamma_{p}}{\Gamma_{i}}
$$

According to this definition, the plasma actuator has an efficiency of the order of $0.1 \%$ (figure 16). This result is in agreement with the experimental investigation by Jolibois \& Moreau (2009) who showed a similar efficiency for a steady forcing. Hence, the present estimation, based on the work by Kriegseis et al. (2011, 2013), indicates that the low efficiency of the plasma actuators erodes the potential gain by the presented control technique. A technical challenge in designing more efficient plasma actuators is to increase the efficiency from $0.1 \%$ to $1 \%$ in order to push $\Gamma$ over the breakeven point. A critical aspect is identified in the zero-forcing voltage $V_{0}$ in (5.2); this offset represents the energy that the plasma actuator needs to create the plasma stream that will cause the force on the flow. This energy is not directly used to control the disturbance in the flow and, hence, it does not contribute to the transition delay.

However, the presented control technique can be generalised to other types of actuators that are able to produce a TS-wave-like disturbance. Examples of this type of actuators can be found in the review by Cattafesta \& Sheplak (2010).

\section{Conclusions}

We have shown that reactive linear adaptive control can efficiently delay the laminar-to-turbulent transition in a realistic low-amplitude disturbance environment. Moreover, it is shown that the drag reduction that results from the transition delay leads to a net power saving up to the order of $10^{3}$, when an ideal-actuator model is considered. The control scheme is able to delay the transition up to an incipient transition occurs at the actuation position. The performance degrades gradually as the amplitude of the perturbation increases. Adaptivity is able to marginally improve the control performances with respect to the nonlinear behaviour of the flow, at least for the investigated set-up.

The large net energy saving shown in an ideal framework highlights the potential performance of reactive transition-delay control. However, in more realistic scenarios, where the actual effort to create a volume forcing inside the boundary layer is taken into account, the energy saving is considerably smaller. When investigating one particular plasma-actuator model (Kriegseis et al. 2011), the energy gain is estimated 
to drop below the break-even point for almost all the investigated cases. The reason for this is be found in the poor efficiency of this particular plasma actuators and therefore an improved actuator design is necessary in order to take advantage of the potential of the investigated control technique.

\section{Acknowledgements}

The authors acknowledge support by the Swedish Research Council (VR-2012-4246, VR-2010-3910) and the Linné Flow Centre. Simulations have been performed at National Supercomputer Centre (NSC) and High Performance Computing Center North (HPC2N) with computer time provided by the Swedish National Infrastructure for Computing (SNIC).

\section{REFERENCES}

Ardekani, I. T. \& Abdulla, W. 2010 Theoretical convergence analysis of fxlms algorithm. Signal Process. 90 (12), 3046-3055.

Aström, K. J. \& Wittenmark, B. 1995 Adaptive Control, 2nd edn. Addison Wesley.

Bagheri, S., Brandt, L. \& Henningson, D. S. 2009 Input-output analysis, model reduction and control of the flat-plate boundary layer. J. Fluid Mech. 620, 263-298.

Belson, B. A., Semeraro, O., Rowley, C. W. \& Henningson, D. S. 2013 Feedback control of instabilities in the two-dimensional Blasius boundary layer: the role of sensors and actuators. Phys. Fluids 25, 054106.

Cattafesta, L. N. \& Sheplak, M. 2010 Actuators for Active Flow Control. Annu. Rev. Fluid Mech. 43, 247-272.

Chevalier, M., Schlatter, P., Lundbladh, A. \& Henningson, D. S. 2007 A pseudo-spectral solver for incompressible boundary layer flows. Tech. Rep. TRITA-MEK 2007:07. KTH Mechanics, Stockholm, Sweden.

Dadfar, R., Fabbiane, N., Bagheri, S. \& Henningson, D. S. 2014 Centralised versus decentralised active control of boundary layer instabilities. Flow Turbul. Combust. 93 (4), $537-553$.

Dadfar, R., Semeraro, O., Hanifi, A. \& Henningson, D. S. 2013 Output feedback control of blasius flow with leading edge using plasma actuator. AIAA J. 51 (9), 2192-2207.

Fabbiane, N., Bagheri, S. \& Henningson, D. S. $2015 a$ Adaptive control of finiteamplitude 3D disturbances in 2D boundary-layer flows. In International Symposium on Turbulence and Shear Flow Phenomena (TSFP-9): http://www.tsfp-conference.org/proceedings/ proceedings-of-tsfp-9-2015-melbourne.html.

Fabbiane, N., Semeraro, O., Bagheri, S.\& Henningson, D. S. 2014 Adaptive and model-based control theory applied to convectively unstable flows. Appl. Mech. Rev. 66 (6), 060801.

Fabbiane, N., Simon, B., Fischer, F., Grundmann, S., Bagheri, S. \& Henningson, D. S. $2015 b$ On the role of adaptivity for robust laminar-flow control. J. Fluid Mech. 767, R1.

Jeong, J. \& Hussain, F. 1995 On the identification of a vortex. J. Fluid Mech. 285, 69-94.

Jolibois, J. \& Moreau, E. 2009 Enhancement of the electromechanical performances of a single dielectric barrier discharge actuator. IEEE Trans. Dielec. Elec. Insul. 16 (3), 758-767.

Juillet, F., MCKeON, B. J. \& SChMid, P. J. 2014 Experimental control of natural perturbations in channel flow. J. Fluid Mech. 752, 296-309.

KaChanov, Y. S. 1994 Physical mechanisms of laminar-boundary-layer transition. Annu. Rev. Fluid Mech. 26 (1), 411-482.

Kotsonis, M., Shukla, R. K. \& Pröbsting, S. 2015 Control of natural Tollmien-Schlichting waves using dielectric barrier discharge plasma actuators. Intl J. Flow Control 7 (1-2), 37-54.

Kriegseis, J., Möller, B., Grundmann, S. \& Tropea, C. 2011 Capacitance and power consumption quantification of dielectric barrier discharge (DBD) plasma actuators. J. Electrostat. 69 (4), 302-312. 
Kriegseis, J., Schwarz, C., Tropea, C. \& Grundmann, S. 2013 Velocity-information-based force-term estimation of dielectric-barrier discharge plasma actuators. J. Phys. D 46 (5), 055202.

Kurz, A., Goldin, N., King, R., Tropea, C. D. \& Grundmann, S. 2013 Hybrid transition control approach for plasma actuators. Exp. Fluids 54 (11), 1-4.

LI, Y. \& GASTER, M. 2006 Active control of boundary-layer instabilities. J. Fluid Mech. 550, 185-205.

Nordström, J., Nordin, N. \& Henningson, D. S. 1999 The fringe region technique and the Fourier method used in the direct numerical simulation of spatially evolving viscous flows. SIAM J. Sci. Comput. 20 (4), 1365-1393.

Schlatter, P., Li, Q., Brethouwer, G., Johansson, A. V. \& Henningson, D. S. 2010 Simulations of spatially evolving turbulent boundary layers up to $R e_{\theta}=4300$. Intl J. Heat Fluid Flow 31 (3), 251-261.

Schlatter, P., Stolz, S. \& Kleiser, L. 2004 LES of transitional flows using the approximate deconvolution model. Intl J. Heat Fluid Flow 25 (3), 549-558.

Schultz-Grunow, F. 1940 Neues Widerstandgesetz für glatte Flatten. Luftfahrtforsch 17, 239.

Semeraro, O., Bagheri, S., Brandt, L. \& Henningson, D. S. 2013 Transition delay in a boundary layer flow using active control. J. Fluid Mech. 731, 288-311.

Simon, B., Nemitz, T., Rohlfing, J., Fischer, F., Mayer, D. \& Grundmann, S. 2015 Active flow control of laminar boundary layers for variable flow conditions. Intl J. Heat Fluid Flow 56, 344-354.

SNyder, S. D. \& HANSEN, C. H. 1994 The effect of transfer function estimation errors on the filtered-x LMS algorithm. IEEE Trans. Signal Process. 42 (4), 950-953.

Stroh, A., Frohnapfel, B. \& Schlatter, P. 2015 A comparison of opposition control in turbulent boundary layer and turbulent channel flow. Phys. Fluids 27, 075101.

Sturzebecher, D. \& Nitsche, W. 2003 Active cancellation of Tollmien-Schlichting instabilities on a wing using multi-channel sensor actuator systems. Intl J. Heat Fluid Flow 24, 572-583. 